\title{
Adjusting the Drafter for COVID19: Re-designing our society's understanding of misinformation
}

\author{
Ajay Agarwal ${ }^{1}$ \\ ${ }^{1}$ DIT University, Dehradun, India \\ verslinfiniaudela@gmail.com
}

\begin{abstract}
The pandemic of COVID19 illuminated the presence of our society's cognition in a lowceiling, inhabitable room, with almost little to no illumination of truth. Such a low-ceiling doesn't only restrict the freedom of our cognition but also inhibits its healthy growth. Subsequently, our society feels a pushing sense, which is often exaggerated by the dark periods of misinformation, disinformation, and fake news. Hence, it becomes essential to rethink the interior designs of our cognition - How can we look at these periods of misinformation from a different lens? Can we use them to our advantage to make our room looks spacious enough for the growth of our cognition? Despite the limitations imposed to the

ceiling length by our existing cognitive biases, there exist multiple, unconventional interdisciplinary approaches from the fields of epistemology, phenomenology, evolutionary psychology, and finally, the mathematics that we, as researchers, can leverage to broaden our understanding of the existing "misinfodemic" that presents as a ripple effect of COVID19 on our society's cognition. The aim of this paper shall be the same - to present a noble discourse regarding the "dark period of misinformation" - why misinformation is NOT a pandemic but a widely-used misnomer, how the source of truthful information acts as a source of misinformation, why misinformation is needed for the development of a better cognitive heuristic framework for our society, and finally, why such unconventional approaches fail to see the light of research. While the existing approaches to deal with misinformation spiral around machine-learning models competing with each other for better detection accuracy, this paper will take the reader right to the epicenter of "misinfodemic" using a variety of routes. Towards the end, the author provides how the mentioned approaches not only widen our understanding regarding the universal phenomenon of misinformation but also can be leveraged and scaled for irrational human behaviors like suicide, partisanship, and even student gun violence in the USA.
\end{abstract}

Keywords: misinformation; psychology; interdisciplinary research; society; evolution 


\section{COVID19, Misinformation, and Society}

Since the start of 2020, our world has been involuntarily subjected to various operational dissections that have exposed the cracks and crevices in sociological, psychological, and medical architecture. As the world struggles with COVID19, the psycho-social comorbidities that generate a ripple effect atrociously damage our current cognition and decision-making skills. One of the most infectious and communicable comorbidities is misinformation. [1-3].

Misinformation, along with its misnomers commonly prevalent among the society, has posed a challenge to our society whose impact and consequence are equivalent to the pandemic scale. UN and other internationally recognized organizations, along with world governments have accepted the presence of misinfodemic that our world collectively faces. However, despite our acceptance and recognition of misinformation as a pandemic, we have recursively failed to tackle the challenge even remotely. Credit for continuous failure can be allocated to multiple stakeholders, however, it is important to prioritize solution brainstorming over failure credit allocation.

The most utilized approaches that aim to deal with the problem of misinformation, are mediumcentric, i.e., they aim to detect the virus in the medium that it spreads. In the case of misinformation, this manifests in machine learning-based approaches to detect, annotate, and collect pieces of misinformation that are shared on social media platforms. However, the rise of COVID19 across the globe as potential competition and challenge for our existing ML-based models has clearly defined the definition of true failure. No stochastic machine learning-based approach to date has shown practically acceptable accuracy that can be utilized as an intervention for our efforts. [19-25]. Besides this, misinformation is a medium agnostic, i.e., it does not depend on the medium for its propagation and reproduction. Misinformation is biascentric. It thrives on our existing cognitive biases and our increased susceptibility to them. Therefore, it becomes important to realize that misinfodemic is not a biological phenomenon whose solution stands limited in the area of one particular domain or discipline. Hence, approaches that can prove to be successful towards attaining any progress in tackling the challenge must be collaborative and interdisciplinary. This brings us closer to understanding the nature of this paper.

This paper aims to tackle the challenge of misinformation from the standpoint of socioevolutionary psychology. Socio-evolutionary psychology is an interdisciplinary field devised by the author for explaining sociological anomalies like misinformation as sociological requirements that provide a favorable environment for certain evolutionary psychological changes that developed in the species Homo sapiens over the years. The aim is to explore and possibly prove certain baseline hypotheses regarding the requirements of misinfodemic for favorable selection of sub-species in species Homo sapiens with "higher heuristics embedded with more efficient analytical skills less susceptible towards cognitive biases". 


\section{Susceptibility towards Misinformation}

Unlike COVID19, misinformation preys on the victim's defense against cognitive biases. [45]. One of the most available platforms through which humans contract misinformation is social media. However, being medium-agnostic misinformation preys on our bias and propagates through it. This means that whilst the freely available piece of misinformation exists in the social media sphere of our lives, our choice to believe it and then share it ahead within our network becomes the dangerous point. The instance we consume a piece of misinformation in any shape or form (images, text, video, or other ensembles), and embed it in our belief systems due to its appeal towards our cognitive bias, it is from then that we are infected with the misinfodemic.

Therefore, the question that arises is what are cognitive biases and what biases does misinformation appeal to? Cognitive biases, in the most simplistic forms, are analytical procedures and algorithms that are embedded in the decision-making process that helps us analyze and process large chunks of information and filter the ones to believe, and the ones not to. [6-7]. These biases were developed and embedded throughout evolutionary cycles in our brains. Despite the true intention for filling a required necessity for the human brain, today, in our dynamic and content-frenzy world we are suffering from unrequited amounts of cognitive overload. As a result, the load on our decision-making framework has not only suffered in terms of the nature of cognitive input but also the stakes involved in the process.

Let us take a short use-case to understand this. Health-based misinformation, as shown in Figure 1, has been on the rise since the start of COVID19. Unchecked and unverified posts by alleged doctors and medical professionals have been shared on platforms like Twitter, that provide effective recipes to cure COVID19 in its early as well as late stage.

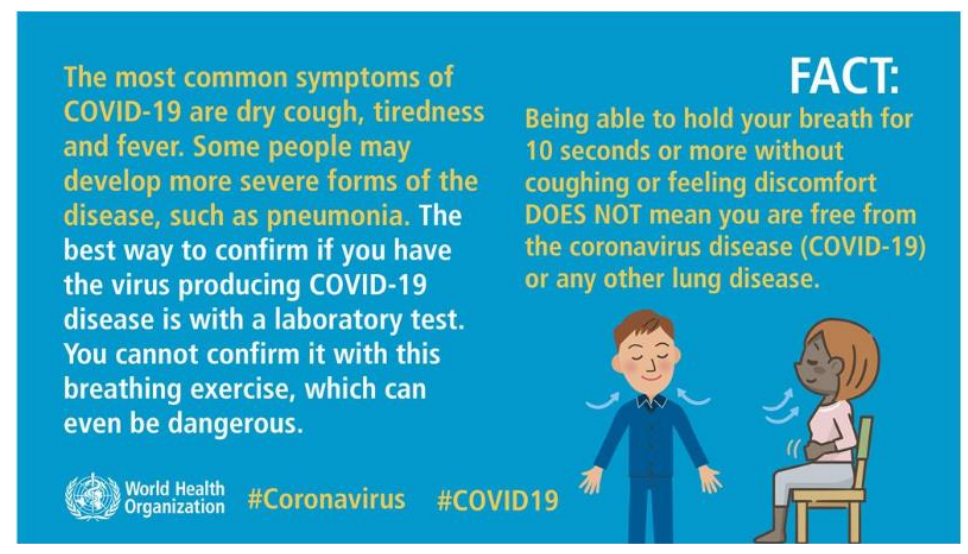

Fig. 1 Myth-buster infographic depicting the true information behind the misinformation has been a frequent activity performed by the United Nations during the pandemic. [8]. This 
highlights the severity and rampant ignorance regarding the public's call for logical analysis for processing new pieces of misinformation.

Let us take a sample tweet that claims to provide a cure for COVID19 through a home-made recipe. A person named $\mathrm{ABC}$ founds this post on his Twitter feed. He reads it. Till this instance, the condition is under control. However, if at this moment, his decision-making framework fails to identify this cognitive input as hogwash, and embeds it in his sub-conscious belief system, he would be infected by misinfodemic. The stakes of believing in a piece of misinformation may not be visible in plain sight, however, are silent digressions from normal human behavior. These include increased vulnerability to future waves of misinfodemic, increased impulsiveness in the decision-making process, and increased susceptibility towards other psychological disorders. The belief in a recipe to cure COVID19 is likely to create an intent for action to try the recipe, which in turn shall elicit a negative response on its observed failure, or a delusional response to ignore its observed failure. Both sides are harmful enough and can severely hinder our psycho-emotional homeostasis. This collective process has been aptly depicted in Figure 2.

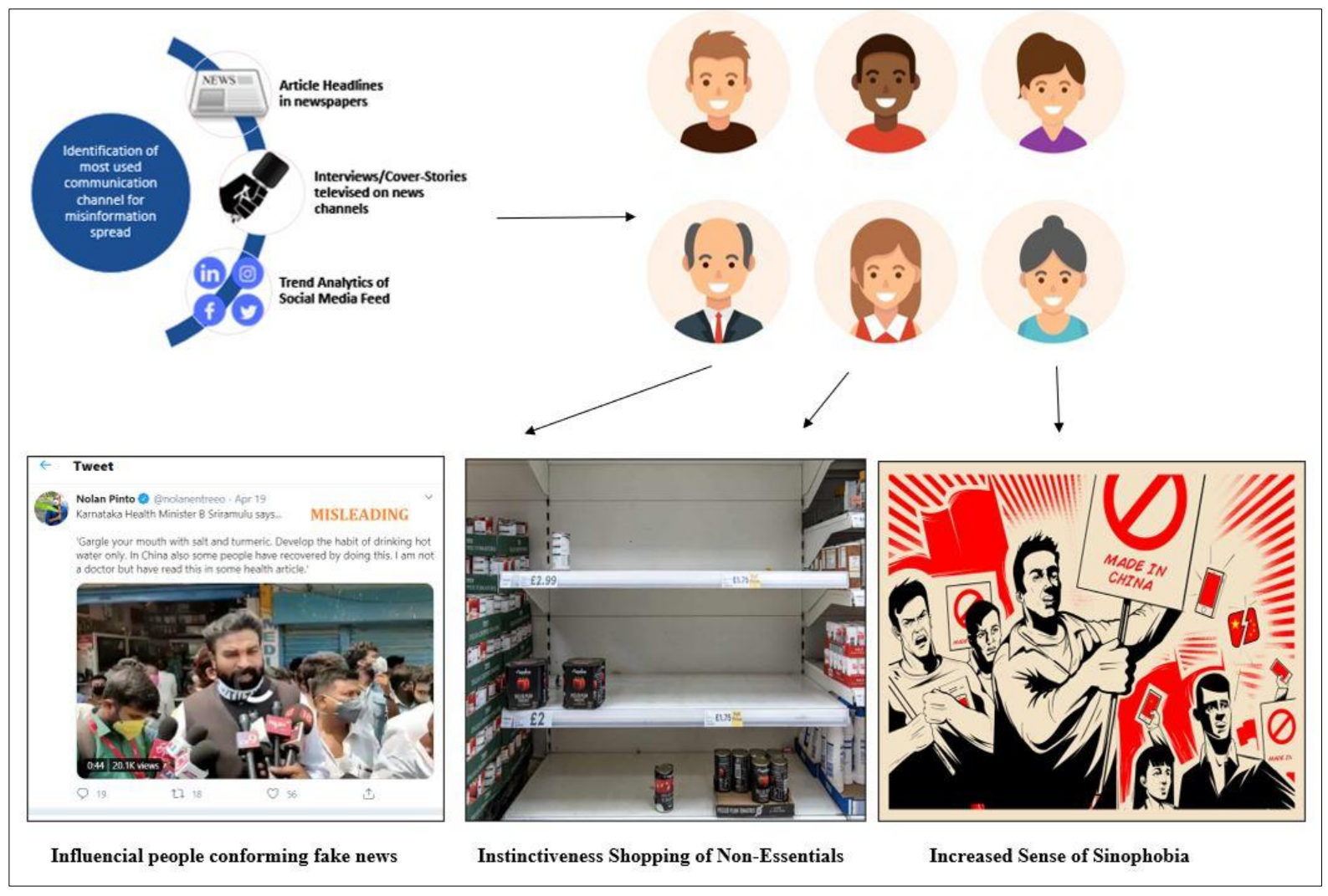

Fig. 2 The above figure is a rendition of the vicious cycle that continues during a healthbased misinfodemic. Notice that at the start of this cycle are the outlets of cognitive input, like newspapers, television channels, and social media that barrage a load of misinformation regarding COVID19 on to different sections of society. In extreme conditions of rampantly believing misinformation and rapid contraction with the misinfodemic, results as drastic and illogical can arise, like - public figures conforming fake news leading to increased fears and 
misinfodemic susceptibility, instinctive shopping of non-essential goods, and finally international hate. [9-12].

With the pressure points established, let us move ahead and understand the prevalent factors that misinformation appeals to, within the aspect of social media. A recent study conducted by NUS; Singapore identified certain key factors that play a major role in an individual's decisionmaking process for associating belief with a piece of misinformation found online. [18]. These include - individual's limited time, amount of content, the complexity of the topic, perceived trustworthiness of the source, the attractiveness of content, experience, and public reaction to the content. These are just a list of the most contributing factors towards the process, apart from the pre-existing cognitive biases. As depicted in Figures $3 \& 4$, it must be realized how a single piece of misinformation on social media itself entails such an exaggerated attack mechanism that digresses our decision-making capacity for the worse - hence resulting in us believing in the piece of misinformation.
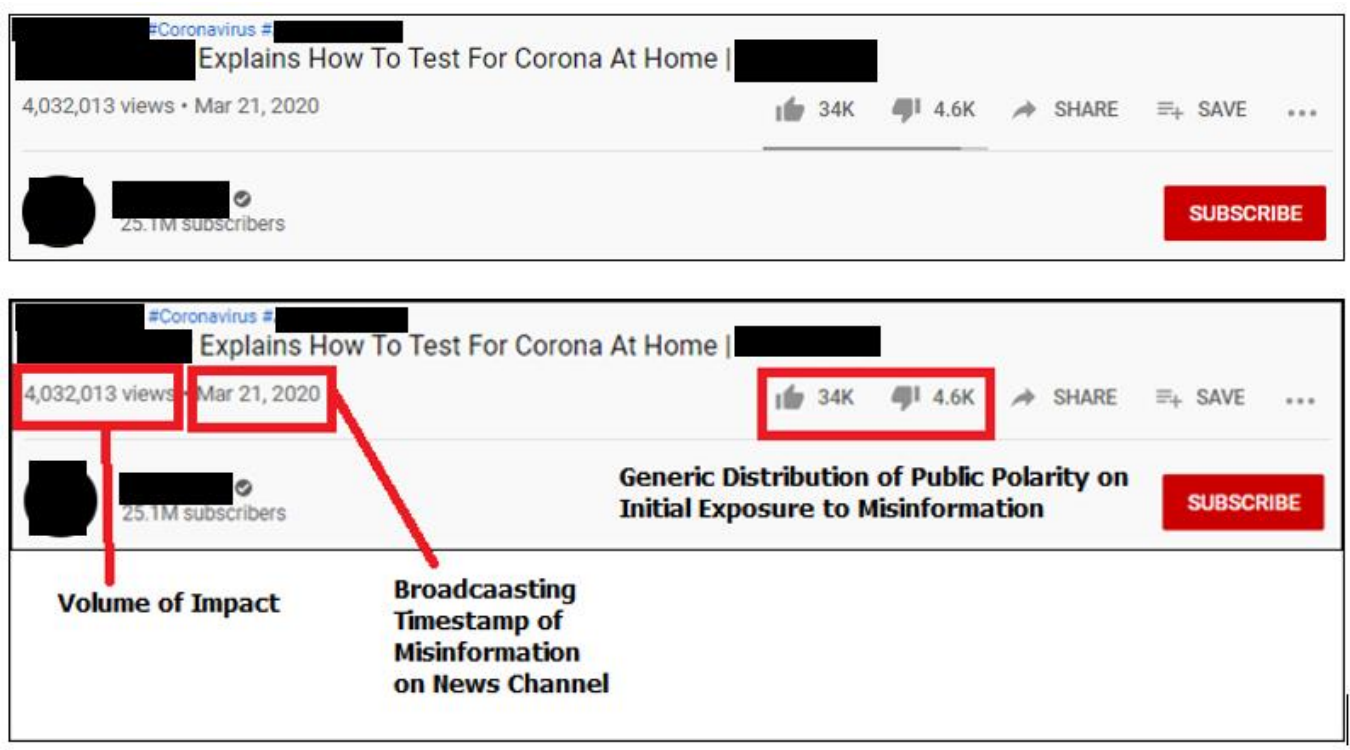

Fig. 3 An example of cognitive analysis of a YouTube header for a misinformation video aired by a news channel (Names and identities are not displayed here to protect the privacy of the user). 


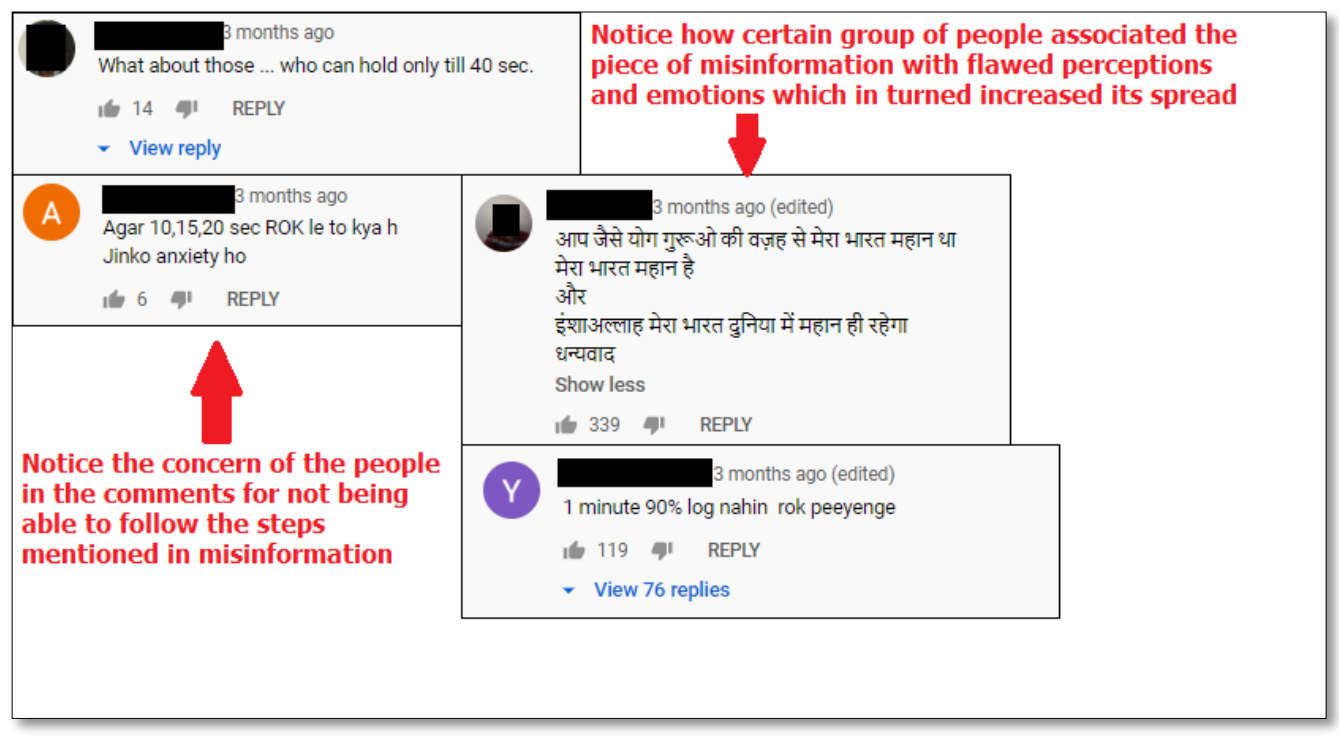

Fig. 4 Random comments are taken from the above misinformation piece aired on the news broadcasting channel. Notice the general public sentiment and how easily a simple piece of misinformation has elicited strong emotions of regret for not being able to follow along with the misinformed procedure. (Names and identities are not displayed here to protect the privacy of the user).

Given the key factors that contribute towards the environment that empowers our own cognitive biases and inherently weakens our decision-making capacity, let us now learn about some of the most contributing cognitive biases that increase our susceptibility to misinfodemic.

a. False Consensus Effect - People believe in false information when a significant number of others appear to believe in it as well.

b. Worldview Backfire Effect - People's belief in false information increases when a correction (to the false information) intentionally or unintentionally also challenges their worldview.

c. Overkill Backfire Effect - People's belief in false information increase when processing a correction (to the false information) requires more cognitive effort than just believing the false one.

d. Illusory Truth Effect - Increase exposure and familiarity with false information increases peoples' likelihood to accept it as true.

e. Motivated Reasoning Bias - People tend to embrace information as true which stands consistent with their pre-existing beliefs and often rejects information as false that contradicts them.

The above list aptly summarizes various cognitive biases that contribute the most towards our contraction with misinfodemic. It is now important to understand how our existing decisionmaking process deals with these biases, and how this research fits in the process. 


\section{Bias and Kahneman's Decision Making}

In the above section, we realized the influence of our inherent cognitive biases in the decisionmaking process. However, the most obvious question that arises is how does our analytical framework deal with the same, especially when it is so prone to making errors? Also, is misinformation the only type of cognitive input we err, or the list extends beyond?

To get a better insight into the human's decision-making process, we refer to Kahneman's dual cognition work. Kahneman has argued in his works about the presence of a dual-cognition system that builds the framework of our decision-making process. [13-15]. These duals systems are labeled as - System 1 and System 2. System 1 acts as the first 'line of defense against any input, and historically performs the cognitive processing. The heuristic tools of cognitive biases are reported to reside in System 1. As a result, System 1 results for any decision, especially associating trust with any new piece of information are highly biased and commonly fooled due to the existing notions, preconceived beliefs, and the spectrum of cognitive biases. This is where System 2 comes into action. System 2 asserts 'executive control' over the decisions made by System 1. System 2 is more analytical and is free from the influence of any cognitive biases, and therefore acts as the "last line of defense" before a final decision is made and acted on by the human (or stored in subconscious memory).

However, despite the presence of such dual cognition, it has been empirically and experimentally found that System 2 is lazy and slow. Therefore, it often overrides and defaults to the decision made by System 1, without any analysis and logical reasoning. Consequently, a significant contribution towards our established decision and beliefs is basically (and literally) "the first un-analyzed thought that comes in our mind". While the degree of activeness for System 1 and System 2 may vary greatly from person to person, a simple cognitive test was created by Frederick; called "Frederick's CRT." [16-17].

"CRT is designed to measure an individual's tendency to override a prepotent response (as provided by our System 1 subject to various cognitive bias) and engage in further reflection (elicit activity from System 2) that leads to a correct response."

The existing literature on decision making and analytical thinking has been established, with sufficient evidence, regarding the empirical validity of Kahneman's System 1 and System 2 thinking model, and Frederick's CRT in checking System 2 engagement. The question that, therefore, arises is how this research fits in the model and what hypothesis does it hope to establish using its research methodology. The next section shall establish the answers to these questions. 


\section{Hypothesis and Methodology}

This paper aims to understand misinfodemic as a universal phenomenon that has arisen in form of a socio-evolutionary filter to segregate sub-groups among the human species which have a "better heuristic and analytical framework" i.e., these individuals have a more active System 2 than System 1, and can analytically engage with the increased cognitive overload that the species (Homo sapiens) face in the coming times.

For this purpose, a self-designed survey was introduced - called the MMBT.

Multimodal Misinformation Belief Test (MMBT) is a survey conducted (with credential/respondent ID) to check the level of trust in news sources of misinformation of multi-modal nature (Text, Image, Video, and Binary Hybrid). MBBT is a one-of-its-kind belief test designed to efficiently and quickly categorize people based on their initial exposure to pieces of misinformation of varying nature. MMBT has two components - one being the multimodal misinformation belief survey, and the second is the Frederick CRT. The reason for including the Frederick CRT in MMBT was to measure the respondent's difference between his/her cognitive processing and validate the hypothesis against a universal score for cognitive bias assessment.

As per the aim, the null hypothesis for MMBT translates into the following two points -

1. Respondents who show a lower belief on pieces of misinformation have a higher score on the CRT test, i.e., System 2 is more active/conscious for people with a trust score < 50 in the MMBT test.

2. Respondents who show a higher belief on pieces of misinformation have a lower score on the CRT test, i.e., System 2 is less active/conscious for people with a trust score > 50 in the MMBT test.

The role of distribution and conduction of bias-free survey was assigned to third-party services that provide these services for free. A randomized sample size of 1000 candidates was taken within the age group of 18 to 50. These respondents belonged from various states in India. The sample size was restricted to the country India due to the current manifestations in local freedom for researchers due to the nationwide implemented non-pharmaceutical interventions. For the future stage in this research, the sample size could be increased and diversified to include groups from different countries as well. Also, no retesting was performed, as the aim of the MMBT is to measure the belief scores on initial exposure, and retesting might comprise the neutrality of the initial exposure scores. The methodology for the survey has been summarized in Figure 5 for a better understanding of the reader. 


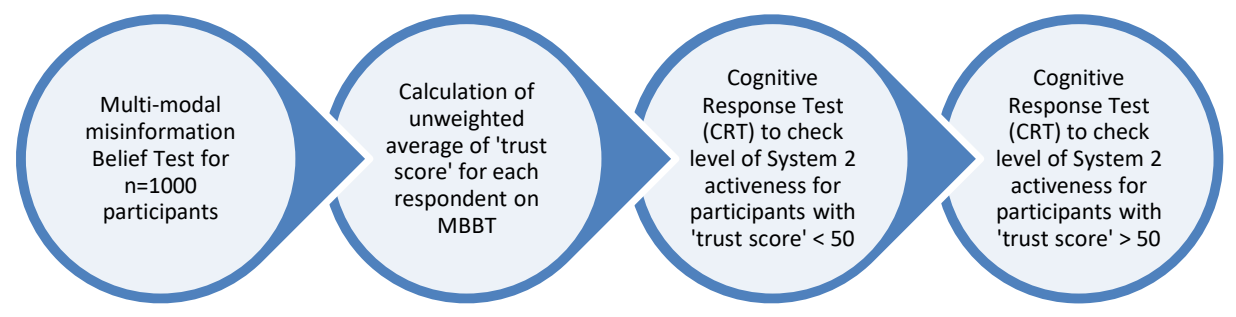

Fig. 5 The above flowchart depicts and summarizes the methodology to be adopted for the conduction of the MMBT test.

\section{Results}

The original Friedrick's CRT consists of only three logical questions. Hence, to measure the validity of our null hypothesis, we can divide our observations primarily into two groups - one, who passed Frederick's CRT (scored 2 or above in CRT), and the last, who failed Frederick's CRT (scored less than 2). Consequently, the mathematical depiction for our observations for proving our null hypothesis becomes that - the first group must have the MMBT score less than or equal to 50, whereas the second group must have their MMBT score more than 50 .

\section{SAMPLE SIZE ( $\mathrm{N=1000)}$ DISTRIBUTION BASED ON TOTAL OUTCOME ON FREDERICK'S CRT}

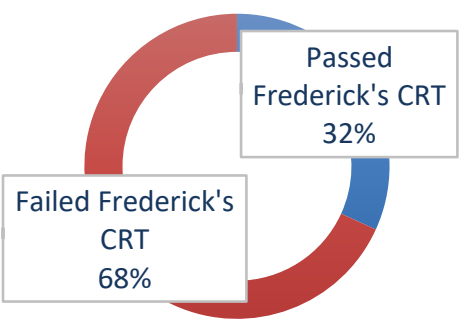

- Passed Frederick's CRT — Failed Frederick's CRT

Fig. 6 The above pie-chart representation depicts the distribution of the sample size $(n=1000)$ based on their results in Frederick's CRT. It was found that 682 candidates failed the CRT (scored less than 2), and 318 candidates passed the CRT. 


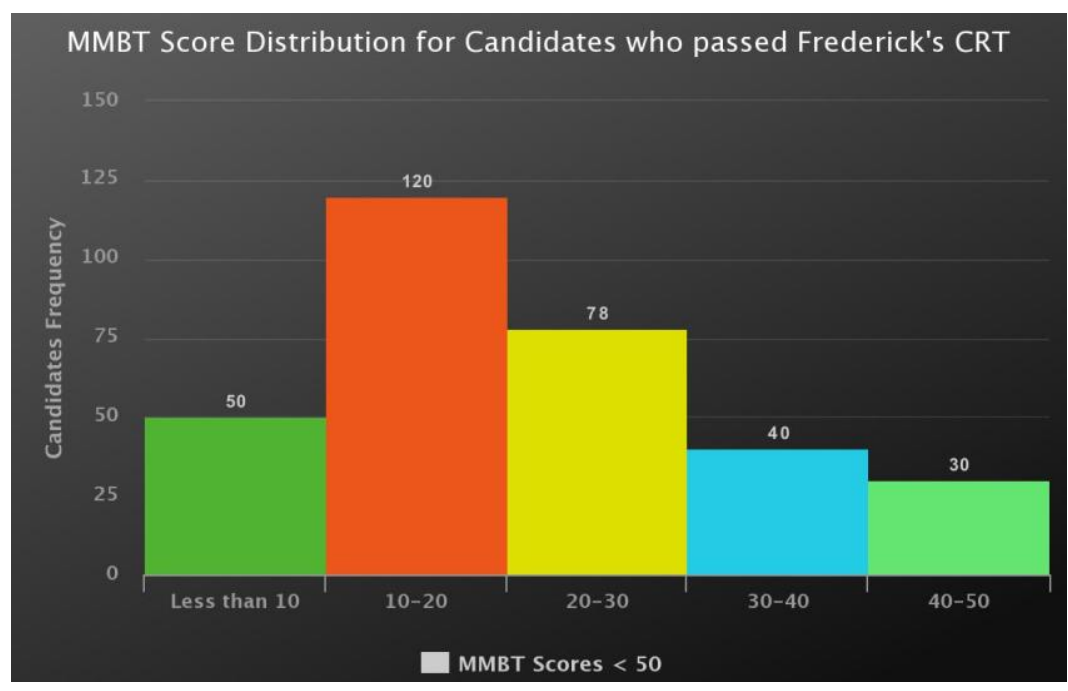

Fig. 7 The above histogram representation depicts the MMBT score distribution for the 318 candidates that passed Frederick's CRT. Notice, the average MMBT score for the group peaks around in the range of 10-20 which in turn represents their active System 2 (that doesn't override System 1 on initial exposure to misinformation).

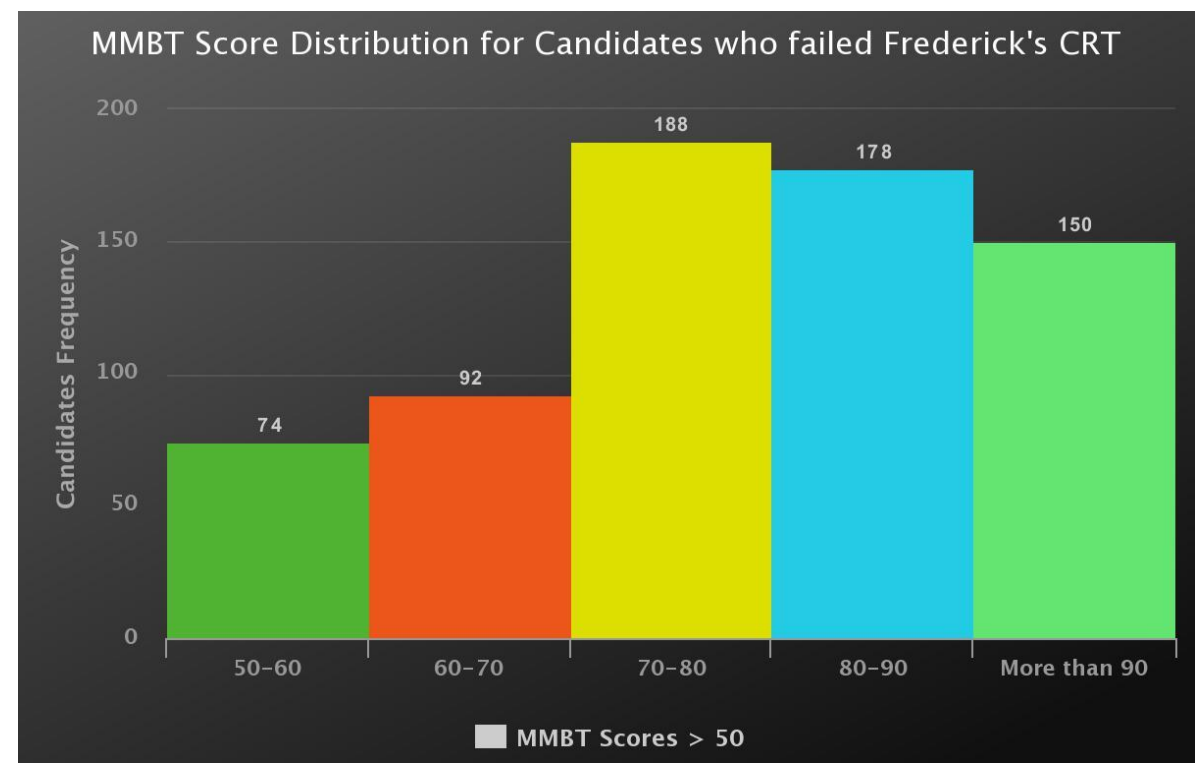

Fig. 8 The above histogram representation depicts the MMBT score distribution for the 682 candidates that failed Frederick's CRT. Notice, the average MMBT score for the group peaks around in the range of 70-80 which in turn represents their less-active System 2 (that does override System 1 on initial exposure to misinformation).

Figures 6-8 depict the results and statistical inferences that we obtained from the conduction of the MMBT. Based on the statistical visualization of our results, we can clearly say that the null hypothesis for our survey has been validated. It is indeed that people who easily fall for their cognitive biases, as a result of less active System 2, associate higher belief with misinformation on its initial exposure, as compared to their counterparts. 
However, it must be noted that such a research methodology needs a greater deal of validation by ensemble approaches and larger sample sizes, to fully validate the null hypothesis for the entire species Homo sapiens. Due to the current restrictions imposed due to COVID19, the possibility of conducting a full-scale cross-sectional survey is highly restricted.

\section{Conclusion and Future Work}

The study proves the validity of the socio-evolutionary psychology approach towards understanding the problem of misinformation. This study, in its root form, provides a central baseline that can be utilized by researchers to come for understanding misinformation as a socio-evolutionary necessity in the world for filtering and providing an evolutionary kickstart for the genesis of more active System 2 in the Homo sapien species. Not only this, but the study also provides and highlights the necessity for a collective, and collaborative need for interdisciplinary approaches to visualize the problem of misinformation, Unlike COVID19, misinformation is not a new phenomenon; rather is a recurring event in our society with no foreseeable cure. Also, in contrast to COVID19, however, misinformation stands on the lighter and easier side for detection and diagnosis and does not entail asymptomatic and symptomatic variations as associated with other biological pandemics. Thereby, the symptoms of misinfodemic infection have been aptly highlighted in Figure 9.

We, as researchers, must realize that misinformation is not a biological entity. Rather, it is a cross-disciplinary one. Hence, it is only when we extend our boundaries across the established definitions of research disciplines and approaches for tackling unexplained sociological phenomenon, can we truly understand the topology of misinformation and successfully discover its anti-particle. 


\section{Signs of Misinfodemic Infection}

\author{
1. Inability to \\ stay away from \\ news streams \\ regarding \\ COVIDI9
}
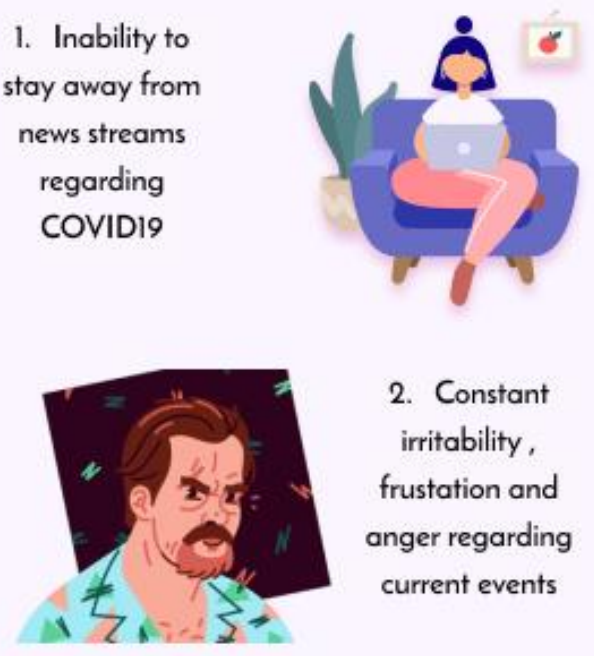

2. Constant irritability, frustation and anger regarding current events

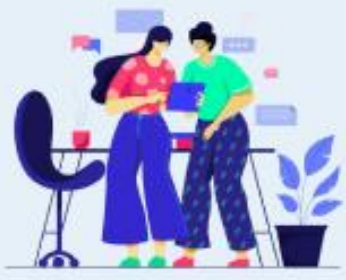

3. Easily believing any piece of COVIDI9 related information on social media

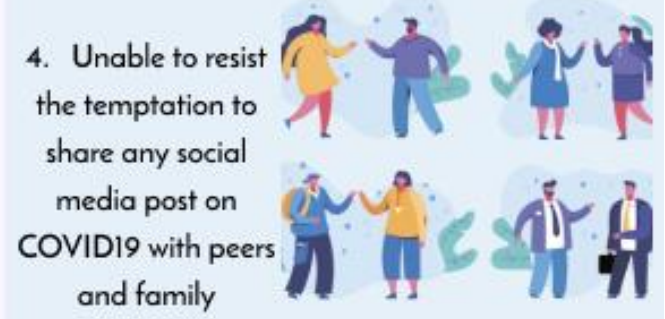

Fig. 9 The above figure represents the author's rendition of the symptoms and signs of misinfodemic infection in an individual. These symptoms were collected based on an exhaustive literature review conducted during this research.

\section{Compliance with Ethical Standards}

1. The author declared no conflict of interest.

2. This article does not contain any studies with human participants or animals performed by the author.

3. No funding in any form or manner was obtained for the conduction of this study. 


\section{References}

[1] Cuan-Baltazar, J. Y., Muñoz-Perez, M. J., Robledo-Vega, C., Pérez-Zepeda, M. F., \& Soto-Vega, E. (2020). Misinformation of COVID-19 on the internet: infodemiology study. JMIR public health and surveillance, 6(2), e18444.

[2] Tasnim, S., Hossain, M. M., \& Mazumder, H. (2020). Impact of rumors and misinformation on COVID19 in social media. Journal of preventive medicine and public health, 53(3), 171-174.

[3] Gupta, L., Gasparyan, A. Y., Misra, D. P., Agarwal, V., Zimba, O., \& Yessirkepov, M. (2020). Information and misinformation on COVID-19: a cross-sectional survey study. Journal of Korean medical science, 35(27).

[4] Trethewey, S. P. (2019). Medical misinformation on social media: cognitive bias, Pseudo-Peer review, and the good intentions hypothesis. Circulation, 140(14), 1131-1133.

[5] Acerbi, A. (2019). Cognitive attraction and online misinformation. Palgrave Communications, 5(1), 17.

[6] Caverni, J. P., Fabre, J. M., \& Gonzalez, M. (Eds.). (1990). Cognitive biases. Elsevier.

[7] Trout, J. D. (2005). Paternalism and cognitive bias. Law and Philosophy, 24(4), 393-434.

[8] 5 ways the UN is fighting 'infodemic' of misinformation. (n.d.). Retrieved October 06, 2020, from https://www.un.org/en/un-coronavirus-communications-team/five-ways-united-nations-fighting$\%$ E2\%80\%98infodemic\%E2\%80\%99-misinformation

[9] Brennen, J. S., Simon, F., Howard, P. N., \& Nielsen, R. K. (2020). Types, sources, and claims of Covid19 misinformation. Reuters Institute, 7, 3-1.

[10] No, gargling with turmeric water isn't a cure for COVID-19. (2020, April 26). Retrieved October 06, 2020, from https://www.ha-asia.com/no-gargling-with-turmeric-water-isnt-a-cure-for-covid-19/

[11] Pidd, H. (2020, March 08). UK supermarkets ration toilet paper to prevent stockpiling. Retrieved October 06, 2020, from https://www.theguardian.com/world/2020/mar/08/coronavirus-stockpilingsupermarkets-toilet-paper-hand-gel

[12] Schild, L., Ling, C., Blackburn, J., Stringhini, G., Zhang, Y., \& Zannettou, S. (2020). " go eat a bat, chang!": An early look on the emergence of sinophobic behavior on web communities in the face of covid-19. arXiv preprint arXiv:2004.04046.

[13] Kahneman, D. (2017). Thinking, fast and slow.

[14] Kahneman, D., \& Tversky, A. (2013). Choices, values, and frames. In Handbook of the fundamentals of financial decision making: Part I (pp. 269-278).

[15] Tversky, A., \& Kahneman, D. (1974). Judgment under uncertainty: Heuristics and biases. science, 185(4157), 1124-1131.

[16] Frederick, S. (2005). Cognitive reflection and decision making. Journal of Economic perspectives, 19(4), 25-42.

[17] Oechssler, J., Roider, A., \& Schmitz, P. W. (2009). Cognitive abilities and behavioral biases. Journal of Economic Behavior \& Organization, 72(1), 147-152.

[18] Soon, C., \& Goh, S. (2018). Fake news, false information and more: Countering human biases. Institute of Policy Studies (IPS) Working Papers, (31).

[19] Yadav 21/04/2020, R., Yadav, R., Rohit Yadav Rohit is a technology journalist and technophile who likes to..., Rohit Yadav Rohit is a technology journalist and technophile who likes to communicate the latest trends around cutting-edge technologies in a way that is straightforward to assimilate. In a nutshell, \& Yadav, R. (2020, April 26). Has AI Failed Us During This Crisis? Retrieved October 06, 2020, from https://analyticsindiamag.com/has-ai-failed-us-during-this-crisis/

[20] Schuster, T., Schuster, R., Shah, D. J., \& Barzilay, R. (2020). The Limitations of Stylometry for Detecting Machine-Generated Fake News. Computational Linguistics, 1-12.

[21] Thorne, J., Vlachos, A., Christodoulopoulos, C., \& Mittal, A. (2020, January). Evaluating adversarial attacks against multiple fact verification systems. Association for Computational Linguistics.

[22] Schuster, T., Shah, D. J., Yeo, Y. J. S., Filizzola, D., Santus, E., \& Barzilay, R. (2019). Towards debiasing fact verification models. arXiv preprint arXiv:1908.05267. 
[23] Schuster, T., Schuster, R., Shah, D. J., \& Barzilay, R. (2019). Are we safe yet? the limitations of distributional features for fake news detection. arXiv preprint arXiv:1908.09805.

[24] Purian, R., Ho, S. M., \& Te'Eni, D. (2020). Resilience of Society to Recognize Disinformation: Human and/or Machine Intelligence.

[25] Atanasova, P., Wright, D., \& Augenstein, I. (2020). Generating Label Cohesive and Well-Formed Adversarial Claims. arXiv preprint arXiv:2009.08205. 